\title{
Some Properties of a Family of Curves on a Surface.
}

\author{
By C. E. Weatherbers.
}

(Received 4th October 1927. Read 4th November 1927.)

This paper shows briefly how, for a singly infinite family of curves on a given surface, the fundamental properties of the family at any point are associated with three central conics determined by the curves, in a manner resembling that in which the curvature properties of a surface at any point are associated with Dupin's indicatrix. The differential invariants employed are the two-parametric invariants ${ }^{1}$ for the given surface.

1. First Conic. Tendency.

Let $t$ be the unit tangent to the curve at any point $P$ of the surface. This vector is a point-function for the surface. Its derivative in the direction of the unit vector $a$, tangential to the surface, is given by $a \cdot \nabla t$. The resolved part of this derivative in the direction of a we shall call the tendency of the family in that direction. It has the value a. $\nabla \mathbf{t} \cdot \mathbf{a}$. From the definition ${ }^{2}$ of div $t$ it then follows that:

The sum of the tendencies for any two perpendicular directions at a point is invariant, and equal to the divergence of the family of curves (div t) at that point.

If now we introduce the conic

$$
\mathbf{r} \cdot \nabla \mathbf{t} \cdot \mathbf{r}=1
$$

whose centre is at the point $P$, and whose plane is the tangent plane at that point, it is clear that the tendency in any direction varies inversely as the square of the radius of the conic in that direction, having the value zero for the directions of the asymptotes. For the direction inclined at an angle $\phi$ to a principal axis of the conic, the tendency $T$ is given by

$$
T=T_{1} \cos ^{2} \phi+T_{2} \sin ^{2} \phi
$$

where $T_{1}$ is the tendency in the direction of the above axis, and $T_{2}$ that for the perpendicular direction. These two quantities are of opposite signs, and their sum has the value div $t$. 
One of the asymptotes of the conic (1) is the tangent to the curve at the point $P$. For, since $\mathrm{t}$ is a unit vector, it is perpendicular to its derivative $t . \nabla t$. Thus the tendency in the direction of the tangent is zero, and consequently that in the perpendicular direction is div $t$. The two directions of zero tendency will be at right angles provided div $t=0$. Thus:

The locus of the points at which the two directions of zero tendency are perpendicular is the line of striction ${ }^{3}$ of the family of curves.

The direction of the other asymptote, and another expression for the tendency in any direction, may be found as follows. Consider the direction making an angle $\theta$ with that of $t$. The unit vector in this direction is $\mathrm{t} \cos \theta+\mathrm{m} \sin \theta$, where $\mathrm{m}$ is the unit tangent to the orthogonal trajectory of the family. The tendency in the above direction is then given by

$$
T=(\mathrm{t} \cos \theta+\mathrm{m} \sin \theta) \cdot \nabla \mathrm{t} \cdot(\mathrm{t} \cos \theta+\mathrm{m} \sin \theta)
$$

or, since $t$ is perpendicular to its derivatives,

$$
\begin{aligned}
T & =\sin \theta[\sin \theta(\mathrm{m} \cdot \nabla \mathrm{t} \cdot \mathrm{m})+\cos \theta(\mathrm{t} \cdot \nabla \mathrm{t} \cdot \mathrm{m}) \\
& =\sin \theta(\sin \theta \operatorname{div} \mathrm{t}-\cos \theta \operatorname{div} \mathrm{m}) \quad \ldots \ldots \ldots \ldots
\end{aligned}
$$

Thus the directions of the asymptotes are given by

$$
\theta=0 \quad \text { and } \quad \tan \theta=\frac{\operatorname{div} m}{\operatorname{div} t}
$$

so that the second asymptote is in the direction of the vector $t \operatorname{div} t+m \operatorname{div} m$, which is the tangential component of the vector curvature of the orthogonal system ${ }^{4}$ of which the given family forms part. And the tangent of the inclination of the two directions of zero tendency is the ratio of the divergence of the family of orthogonal trajectories to that of the given family.

It follows, as found above, that the asymptotes will be perpendicular where divt vanishes-a condition satisfied at all points by a family of parallel curves. ${ }^{5}$ The asymptotes will be coincident where $\operatorname{div} \mathbf{m}$ is zero. But this is the negative of the geodesic curvature ${ }^{6}$ of the family at the point $P$. Hence:

The locus of the points at which the two directions of zero tendency are coincident is the line of normal curvature ${ }^{7}$ of the family of curves,

The two directions are coincident at all points in the case of a a family of geodesics. 


\section{Second Conic. Moment.}

The change $d t$ in the unit tangent to the curves, due to a displacement $d \mathbf{r}$ from $P$ to a neighbouring point $Q$ is given by ${ }^{8}$

$$
d \mathbf{t}=d \mathbf{r} \cdot \nabla \mathbf{t}
$$

The mutual moment of the tangents at $P$ and $Q$ is the resolved part, in the direction of $\mathrm{t}$, of the moment of $\mathrm{t}+d \mathrm{t}$ about $P$. Consequently it has the value

$$
d \mathbf{r} \times(\mathbf{t}+d \mathbf{t}) \cdot \mathbf{t}=d \mathbf{r} \times d \mathbf{t} \cdot \mathbf{t}=d \mathbf{r} \cdot(\nabla \mathbf{t} \times \mathbf{t}) \cdot d \mathbf{r}
$$

in which $\nabla$ is understood to operate only on the vector immediately following it. If $a$ is the unit vector in the direction $P Q$, the quotient of this mutual moment by $(d r)^{2}$ is the function

$$
M=\mathbf{a} \cdot(\nabla \mathbf{t} \times \mathbf{t}) \cdot \mathbf{a}
$$

We shall call this the moment of the family of curves at the point $P$ for the direction of $a$. If then we introduce the conic

$$
\mathbf{r} \cdot(\nabla \mathbf{t} \times \mathbf{t}) \cdot \mathbf{r}=\mathbf{1}
$$

whose centre is at the point $P$, and whose plane is tangential to the surface at $P$, it is clear from (5) that:

The moment of the family for any direction at $P$ varies inversely as the square of the radius of the conic (6) in that direction, having the value zero for the directions of the asymptotes.

For a direction inclined at an angle $\phi$ to a principal axis of the conic the moment is given by

$$
M=M_{1} \cos ^{2} \phi+M_{2} \sin ^{2} \phi
$$

Where $M_{1}$ is the moment for the direction of the above axis, and $M_{2}$ is that for the perpendicular direction, these two quantities having opposite signs. Consequently the sum of the moments in two perpendicular directions at a point is invariant. It may be called the total moment (or briefly the moment) of the family at that point. Now one of the directions of zero moment is that of the vector $t$; for consecutive tangents to a curve intersect each other. The 
moment for the perpendicular direction, as we have shown elsewhere, ${ }^{9}$ has the value $\mathbf{t}$. curl t. Hence:

The sum of the moments for two perpendicular directions at a point is invariant, having the value $\mathrm{t}$. curl $\mathrm{t}$.

If the two directions of zero moment are at right angles, the total moment is zero; and conversely. Thus:

The locus of points at which the two directions of zero moment are perpendicular is the line of zero moment ${ }^{10}$ of the family of curves.

This property holds at all points for a family of lines of curvature.

The direction of the second asymptote of the conic (6), and another expression for the moment in any direction may be found as follows. In the direction, inclined at an angle $\theta$ to the tangent $t$, the unit vector is $\mathrm{t} \cos \theta+\mathrm{m} \sin \theta$; and the moment in this direction is therefore given by

$$
\begin{aligned}
M & =(\mathrm{t} \cos \theta+\mathrm{m} \sin \theta) \cdot(\nabla \mathrm{t} \times \mathrm{t}) \cdot(\mathrm{t} \cos \theta+\mathrm{m} \sin \theta) \\
& =\sin \theta[\sin \theta(\mathrm{m} \cdot \nabla \mathrm{t} \times \mathrm{t} \cdot \mathrm{m})+\cos \theta(\mathrm{t} \cdot \nabla \mathrm{t} \times \mathrm{t} \cdot \mathrm{m})] \\
& =\sin \theta(\sin \theta \mathrm{t} \cdot \operatorname{curl} \mathrm{t}-\cos \theta \mathrm{m} \cdot \operatorname{curl} \mathrm{t}) \ldots \ldots \ldots \ldots
\end{aligned}
$$

Thus the directions of the asymptotes are determined by

$$
\theta=0 \quad \text { and } \quad \tan \theta=\frac{\mathrm{m} \cdot \operatorname{curl} t}{\mathrm{t} \cdot \operatorname{curl} t}
$$

The second of these is the direction conjugate to that of $t$; for it is the direction of the tangential component ${ }^{11}$ of curl $t$. Thus the two directions of zero moment will be coincident if that of $t$ is selfconjugate (or asymptotic). In this case the principal normal to the curve is tangential to the surface. Hence:

The locus of the points at which the two directions of zero moment are coincident is the line of tangential curvature ${ }^{12}$ of the family of curves.

Also since $-m$. curl $t$ is the normal curvature ${ }^{13}$ of the surface in the direction of $t$, that is to say, the curvature of the geodesic tangent, it follows from (9) that:

The tangent of the angle between the two directions of zero moment is minus the ratio of the curvature to the torsion of the geodesic tangent. 
3. Third Conic. Swerve.

Consider again the derivative of $t$ in the direction of the unit vector a. The resolved part of this in the tangential direction perpendicular to $\mathbf{a}$ is $\mathbf{a} \cdot \nabla \mathbf{t} \cdot(\mathbf{n} \times \mathbf{a})$, where $\mathbf{n}$ is the unit normal to the surface. This quantity may be called ${ }^{14}$ the swerve of the family of curves in this direction. Denoting it by $S$ we have

$$
S=\mathbf{a} \cdot(\nabla \mathbf{t} \times \mathbf{n}) \cdot \mathbf{a}
$$

If then we introduce the conic

$$
\mathbf{r} \cdot(\nabla \mathbf{t} \times \mathbf{n}) \cdot \mathbf{r}=1
$$

whose centre is the point $P$, and whose plane is the tangent plane at $P$, it is clear from (10) that the swerve of the family of curves in any direction varies inversely as the square of the radius of the conic (11) in that direction.

For a tangential direction, inclined at an angle $\theta$ to that of $\mathrm{t}$, the value of the swerve is given by

$$
\begin{aligned}
S & =(\mathrm{t} \cos \theta+\mathrm{m} \sin \theta) \cdot(\nabla \mathrm{t} \times \mathrm{n}) \cdot(\mathrm{t} \cos \theta+\mathrm{m} \sin \theta) \\
& =\cos \theta[\cos \theta(\mathrm{t} \cdot \nabla \mathrm{t} \cdot \mathrm{m})+\sin \theta(\mathrm{m} \cdot \nabla \mathrm{t} \cdot \mathrm{m})] \\
& =\cos \theta(\sin \theta \operatorname{div} \mathrm{t}-\cos \theta \operatorname{div} \mathrm{m}) \quad \ldots \ldots \ldots \ldots
\end{aligned}
$$

Thus the asymptotes of the conic (11), or the directions of zero swerve, are determined by

$$
\cos \theta=0 \quad \text { and } \quad \tan \theta=\frac{\operatorname{div} m}{\operatorname{div} t}
$$

The latter coincides with an asymptote of the conic (1). The former is perpendicular to $t$. It follows that the axes of the conic (11) bisect. the angles between those of the conic (1). From (12) it also follows that:

The sum of the swerves in two perpendicular directions at a point is invariant and equal to the geodesic curvature of the family of curves.

The swerve in the direction of $t$ is the geodesic curvature of the curve; and that for the perpendicular direction is zero. The two directions of zero swerve will be at right angles where div $m$ vanishes. They will be coincident where div $t$ is zero. Hence: 
The locus of the points at which the two directions of zero swerve are perpendicular is the line of normal curvature of the family. The locus of the points at which these two directions are coincident is the line of striction of the family of curves.

In a separate paper ${ }^{15}$ the writer has applied the above ideas to the theory of congruences of curves, thereby effecting a considerable extension, and showing that the properties of curvilinear congruences are to a large extent analogous to those of congruences of straight lines.

1 Cf. the author's Differential Geometry. Chap. XII ; or Quar. Journ. of Math., 50 (1925), 230-269.

2 Differential Geometry, Art. 116.

3 Of. The Mathematical Gavette, 13 (Jan. 1926), 2; or Diff. Geom., Art. 126.

4 The Math. Garette, loc. cit., p. 5 ; or Diff. Geom., Art. 125.

5 Diff. Geom, p. 258.

${ }^{6}$ Ibid, Art. 121.

: Ibid., Art. 130.

${ }^{8}$ Ibid., p. 221.

9 Diff. Geom., p. 258.

10 Ibid., Art. 130.

11 Ibid., p. 258.

$1 \div$ Loc. cit.

13 Diff. Geom., p. 250.

14 Neville uses the term "swerve" for a different function. See his Multilinear Functions of Direction, p. 26.

15 “On Curvilinear Congruences," communicated to the Amer. Math, Soc. 